\title{
Neue "Nationale Strategie» schliesst wichtige Lücke
}

\author{
Claudia Kesslera, Felix Mahler \\ a Dr. med., MPH, FMH Prävention und Gesundheitswesen, Mitglied der Projektleitung CardioVasc Suisse; \\ ${ }^{b}$ Prof. Dr. med., FMH für Innere Medizin spez. Angiologie, Präsident CardioVasc Suisse
}

Herz- und Gefässkrankheiten, Hirnschlag und Diabetes tragen heute in der Schweiz führend zur Krankheitslast bei. Sie verursachen vorzeitige Todesfälle und leisten dem stetigen Anstieg der Gesundheitskosten Vorschub. Viele der zugrundeliegenden Risikofaktoren lassen sich durch Prävention und gezielte Früherkennung jedoch wirkungsvoll bekämpfen. Zudem vermag eine bedürfnis- und bedarfsgerechte, patientenzentrierte Gesundheitsversorgung das Leiden der Betroffenen zu reduzieren und deren Lebensqualität zu verbessern.

Das Bundesamt für Gesundheit (BAG) und die Schweizerische Konferenz der kantonalen Gesundheitsdirektorinnen und -direktoren (GDK) begrüssen krankheitsspezifische Strategien als Ergänzung zur «Nationalen Strategie Prävention nichtübertragbarer Krankheiten

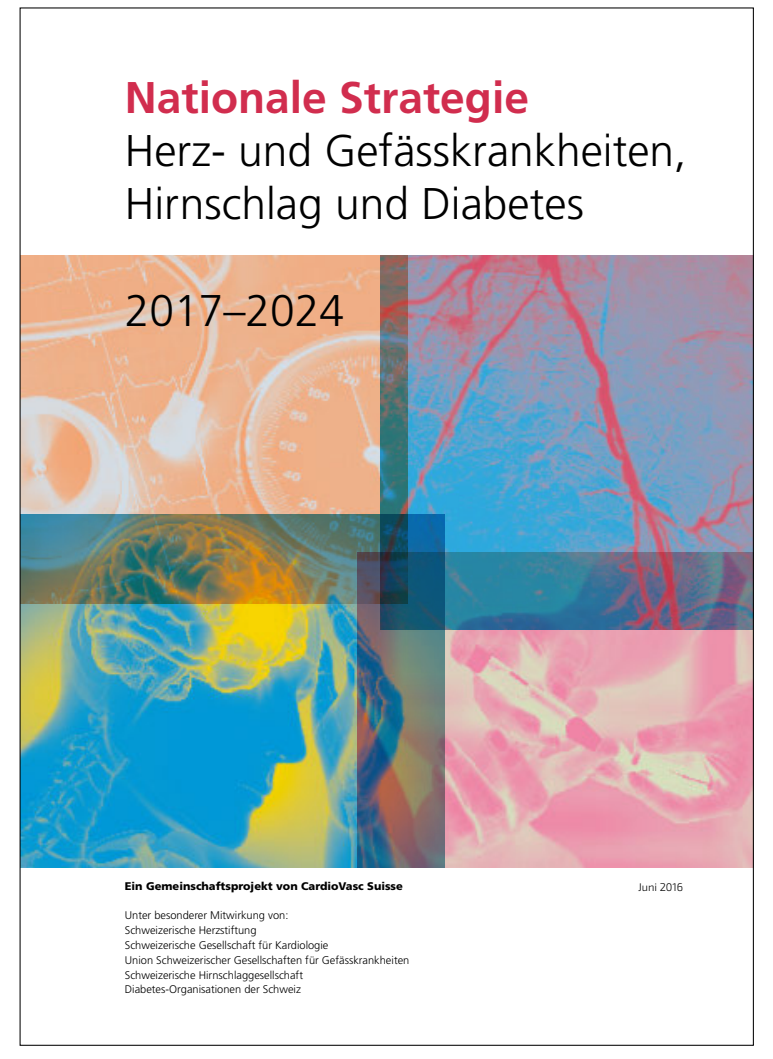

(C) Schweizerische Herzstiftung
2017-2024» (NCD-Strategie). Bisher fehlte für die Schweiz eine spezifische Strategie für Herz-Kreislauf-Erkrankungen und Diabetes. Eine Gruppe von Trägerorganisationen (Schweizerische Herzstiftung, Schweizerische Gesellschaft für Kardiologie, Union Schweizerischer Gesellschaften für Gefässkrankheiten und ihre Mitglieder, Schweizerische Hirnschlaggesellschaft, Diabetes-Organisationen) hat die Initiative ergriffen und schliesst mit der «Nationalen Strategie Herz- und Gefässkrankheiten, Hirnschlag und Diabetes 2017-2024» diese Lücke. Die vorliegende Strategie wurde unter dem Lead des Netzwerks CardioVasc Suisse in einem partizipativen Prozess erarbeitet. Beteiligt waren neben vielen Organisationen der Gesundheitsberufe verschiedener Disziplinen und Professionen auch Patientenorganisationen und andere relevante Akteure.

Die Strategie definiert Prioritäten und schafft für die Akteure einen gemeinsamen Handlungsrahmen. So lassen sich zukünftig Ressourcen besser bündeln und effizienter nutzen, und die politischen Forderungen der Trägerorganisationen gewinnen an Gewicht. In der Praxis der Gesundheitsversorgung soll die Strategie patientenzentriert umgesetzt werden, das heisst, die Bedürfnisse und die oft komplexen Situationen der Betroffenen sollen im Mittelpunkt stehen.

Da die Schweiz heute über eines der höchstentwickelten Gesundheitssysteme verfügt, kann die Strategie auf viel Bestehendem aufbauen. Sie fokussiert deshalb auf die wichtigsten Lücken. Vielfach wurden dabei gemeinsame Anliegen identifiziert, welche für alle beteiligten Akteure einen hohen Stellenwert haben. Die einzelnen Prioritäten der Strategie werden in Kapitel 4 aufgeführt. Sie lassen sich wie folgt zusammenfassen:

\section{Prioritäten der «Nationalen Strategie Herz- und Gefässkrankheiten, Hirnschlag und Diabetes 2017-2024»}

- Prävention und Früherkennung stärken

- Qualitativ hochstehende, integrierte und patientenzentrierte Versorgung von chronisch kranken und multimorbiden Patienten fördern 
- Akutversorgung von Herzinfarkt und Hirnschlag optimieren

- Koordination und Kooperation auf allen Ebenen verstärken

- Datengrundlage für die Schweiz verbessern

- Finanzierungslücken schliessen und gesundheitspolitische Rahmenbedingungen verbessern

Am 13. November 2014 wurde das Projekt und das vorgeschlagene Vorgehen in einem Kick-off Treffen mit den interessierten Stakeholdern diskutiert. Nach einem intensiven partizipativen Entwicklungsprozess wird

\section{Die Strategie definiert Prioritäten und schafft für die Akteure einen gemeinsamen Handlungsrahmen.}

die «Nationale Strategie Herz- und Gefässkrankheiten, Hirnschlag und Diabetes» nun anlässlich eines Präsentationsanlasses am 8. November 2016 in Bern einer breiteren Öffentlichkeit vorgestellt. Die volle Version des Strategiepapiers kann auf Deutsch und Französisch mit folgendem Link heruntergeladen werden: www. cardiovascsuisse.ch/strategie. Zusammenfassungen liegen zusätzlich auch auf Italienisch und Englisch vor. Ohne das grosse Engagement und die konstruktive Zusammenarbeit der beteiligten Personen und Organisationen hätte diese Strategie nicht realisiert werden können. Nun gilt es, die erfolgreich eingeleitete Ko- operation zu vertiefen und auszubauen und über die Zusammenarbeit rund um die vorgeschlagenen Massnahmen das Strategiepapier im Arbeitsalltag umzusetzen, damit bis im Jahre 2024 möglichst grosse Fortschritte im Hinblick auf die strategischen Ziele erzielt werden können.

\section{Autorinnen und Autoren der Strategie}

Dr. med. Claudia Kessler, CardioVasc Suisse (Hauptautorin)

Mitglieder der redaktionellen Kerngruppe (alphabetische Reihenfolge): Andreas Biedermann, MD, CardioVasc Suisse; Therese Junker, Geschäftsführerin Schweizerische Herzstiftung (bis Juni 2016); Prof. Dr. med. René Lerch, Stiftungsrat Schweizerische Herzstiftung; Prof. Dr. med. Felix Mahler, CardioVasc Suisse; Dr. rer. pol. Tania Weng-Bornholt, diabetesschweiz und QualicCare Redaktion der krankheitsgruppenspezifischen Kapitel (alphabetische Reihenfolge): Prof. Dr. med. Peter Diem, Schweizerische Gesellschaft für Endokrinologie und Diabetologie; Prof. Dr. med. Augusto Gallino, Union Schweizerischer Gesellschaften für Gefässkrankheiten; Prof. Dr. med. Philippe Lyrer, Schweizerische Hirnschlaggesellschaft; Dr. med. Gian Marco De Marchis, Schweizerische Hirnschlaggesellschaft; Dr. rer. pol. Tania Weng-Bornholt, diabetesschweiz und QualicCare; Prof. Dr. med. Matthias Wilhelm; Schweizerische Gesellschaft für Kardiologie. 